Please do not remove this page

RMIT

UNIVERSITY

\title{
Microstamp patterning of protein arrays
}

Nasabi, Mahyar; Mitchell, Arnan; Kalantar Zadeh, Kourosh; Nesbitt, Warwick

https://researchrepository.rmit.edu.au/esploro/outputs/9921863878601341/filesAndLinks?institution=61RMIT_INST\&index=null

Nasabi, M., Mitchell, A., Kalantar Zadeh, K., \& Nesbitt, W. (2008). Microstamp patterning of protein arrays. Proceedings of the 2008 International Conference on Nanoscience and Nanotechnology, 117-120.

https://doi.org/10.1109/ICONN.2008.4639260

Published Version: https://doi.org/10.1109/ICONN.2008.4639260

Repository homepage: https://researchrepository.rmit.edu.au

(c) 2008 IEEE. Personal use of this material is permitted. However, permission to reprint/republish this material for advertising or promotional purposes or for creating new collective works for resale or redistribution to servers or lists, or to reuse any copyrighted component of this work in other works must be obtained from the IEEE.

Downloaded On 2023/04/26 23:45:25 +1000 


\title{
Microstamp patterning of Protein Arrays
}

\author{
Mahyar Nasabi ${ }^{1}$, Arnan Mitchell ${ }^{1}$, Kourosh Kalantar-Zadeh ${ }^{1}$, Warwick S. Nesbitt ${ }^{2}$ \\ ${ }^{1}$ School of Electrical and Computer Engineering RMIT University, Australia \\ ${ }^{2}$ The Australian Centre for Blood Diseases, Monash University, Australia \\ Email: Mahyar.Nasabi @student.rmit.edu.au
}

\begin{abstract}
Pathological thrombus formation is initiated by the interaction of blood platelets to immobilized proteins at the vessel wall. Platelet surface adhesion leads to biochemical activation and structural reorganization resulting in spreading of the platelet across the adhesive surface. Extensive studies have been carried out to examine platelet spreading responses on continuous substrates but little is known about the impact of protein surface distribution on platelet function. This paper describes the development of a micro-contact printing technique to establish defined 2-dimensional arrays of the thrombogenic protein fibrinogen at the surface of glass substrates, with the aim of investigating the impact of protein surface distribution on platelet biochemical signaling events associated with the adhesion/spreading process.
\end{abstract}

Keywords: PDMS, Microstamp, Fibrinogen, Platelet.

\section{INTRODUCTION}

Pathological thrombus formation is a complex disease process governed by three fundamental parameters: i) disturbance of haemorheology or blood flow dynamics; ii) abnormalities in blood constituents; and iii) endothelial injury or disruption. Endothelial injury results in the presentation of an array of reactive proteins that both initiate the adhesion of blood platelets to sites of vessel injury and propagate the development of potentially occlusive pathological thrombi. To date, in vitro models of platelet adhesion under both static and blood flow conditions have predominantly utilised purified blood proteins non-specifically adsorbed to glass substrates to investigate platelet adhesion and associated cell signalling events [1-3]. Although useful, these continuous protein substrates do not address the potential effects of non-uniform protein distribution on platelet adhesion and biochemical activation. Given the heterogeneous distribution of adhesive proteins within the blood vessel wall and at sites of vessel injury in vivo, it is of interest to study the interaction of platelets with artificially patterned arrays of adhesive proteins.

Dependent on cell-type and the nature of the protein substrate the interacting cells may undergo a phase of initial adhesion and subsequent spreading. The propensity to spread is in part determined by the separation distance between adjacent protein elements. Thus by carefully controlling the separation and distribution of the protein array, the spatial and temporal dynamics of cell surface interactions can be modulated. Furthermore, the effect of spatially restricting the rate and extent of cell spreading in this way can be utilized to probe the relationships between biochemical signaling events and the structural elements of the cell.
Patterning and attachment of cells to glass has been accomplished by deposition of lipid films with strips of the lipid removed for cell attachment to the clean glass substrates [4]. Metal films can adhere proteins to otherwise non-adhesive surfaces. It is possible to pattern these thin metal films to create protein arrays [5]. Some other methods use surface chemical modification using ultraviolet (UV) irradiation through a mask, allowing dorsal root ganglion (DRG) cells to follow the pattern [6].

One of the most accepted methods over last decade for creating surface patterns is Microstamp printing. Microstamp printing, also known as Micro-contact printing, is well known as an emerging alternative of Photolithography, in which an elastomeric stamp, generally polydimethylsiloxane (PDMS), is used to transfer molecules of the "ink" to the surface of the substrate by contact, like a 'rubber stamp'.

Micro-contact printing of self-assembled monolayers (SAMs) has been reported. Lopez et al. [7] presented patterning the adhesion of mammalian cells to surfaces using SAMs of alkanethiolates on gold substrate. This was then covered with thin layer of protein by exposure of alkanethiols to laminin for selective cell adhesion.

Other, non-biological applications of micro-contact printing have been reported. For instance a self assembled monolayer was printed onto a gold surface and used as ultrathin resists in selective wet etching [8] achieving far higher resolution than is possible with optical lithography. Similarly, a micro-contact patterned self assembled monolayer has been used to control the wetting, dewetting, nucleation, growth, and deposition of other inorganic materials [9].

Many of the biological studies using micro-contact printing to date have been concerned with neural cells and the formation of networks of these cells [10-11]. Neural cells are relatively large, requiring protein islands of the order of $20 \mu \mathrm{m}$ separated by $100 \mu \mathrm{m}$. Micro-contact stamping on this scale is challenging, but has been quite successful. In comparison, discoid blood platelets with a diameter in the order of $2 \mu \mathrm{m}$ are far smaller and thus require islands of $1 \mu \mathrm{m}$ dimension with separation distances in the range of $1-7 \mu \mathrm{m}$. Stamping protein with such high resolution is a significant challenge.

In this paper, we report the production of arrays of the blood protein Fibrinogen using micro-contact printing. The novel aspect of this paper is that we have achieved very high resolution biological patterning with arrays of $1 \mu \mathrm{m}$ features separated by approximately $5 \mu \mathrm{m}$ being demonstrated. 


\section{MATERIALS AND METHODS}

The stamping process is broken into several steps. First an inverse master pattern is fabricated. This master is then cast as a stamp in the material polypolydimethylsiloxane (PDMS). The stamp is surface plasma treated immediately prior to being coated with protein and this protein is printed onto a pretreated microscope slide. Finally, the quality of the stamped protein pattern is assessed by conventional and fluorescence microscopy. Each step is detailed in the following:

\section{A. Master fabrication}

The master pattern was fabricated by coating a glass wafer with a $2.5 \mu \mathrm{m}$ thick film of photoresist, SU-8 2002, and patterning using a photomask with $1 \mu \mathrm{m}$ to $10 \mu \mathrm{m}$ features with various separations ranging from 1 to $10 \mu \mathrm{m}$. The exposed and subsequently thermally cross-linked portions of the film are rendered insoluble to liquid developers. The master was then developed, dissolving the unexposed regions. The resulting master was thus a plane of photoresist patterned with holes (as depicted in Fig 1). The advantages of using SU-8 2002 in compare to positive resist are sturdier mechanical and chemical properties as well as to be capable of producing a high contrast.

\section{B. Stamp fabrication}

The master was coated with a detergent, (5\% Triton-X solution), to aid release of the cast stamp [10]. A cylindrical Perspex shim was placed around the master features of interest (as shown in Fig 1), and was filled with PDMS. A clean glass slide, thinly coated with PDMS, was placed on top of the shim. Both uncured PDMS were placed in a vacuum to remove dissolved gas and avoid bubbles in the final stamp for at least $20 \mathrm{~min}$ before the glass was placed on top of the shim. The stamp was cured at $65^{\circ} \mathrm{C}$ over night. The PDMS adheres strongly to the top glass plate. This provides mechanical support for the stamp. The PDMS was easily removed from both the Perspex shim and the detergent coated master pattern. The resulting stamp was thus a flexible inverse PDMS cast of the master bonded to a rigid glass slide as shown in Fig 1. Fig 2 presents an optical microscope image of a realized stamp.

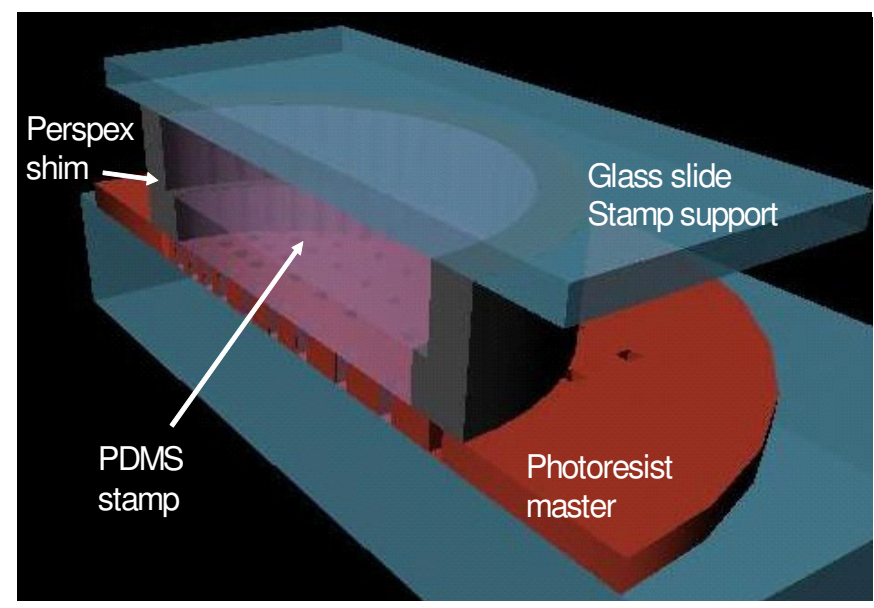

Figure 1. Cross-section of stamp fabrication. The photoresist master is patterned with holes, a perspex shim contains the PDMS stamp during curing.

A glass slide adhered to the stamp provides mechanical support.

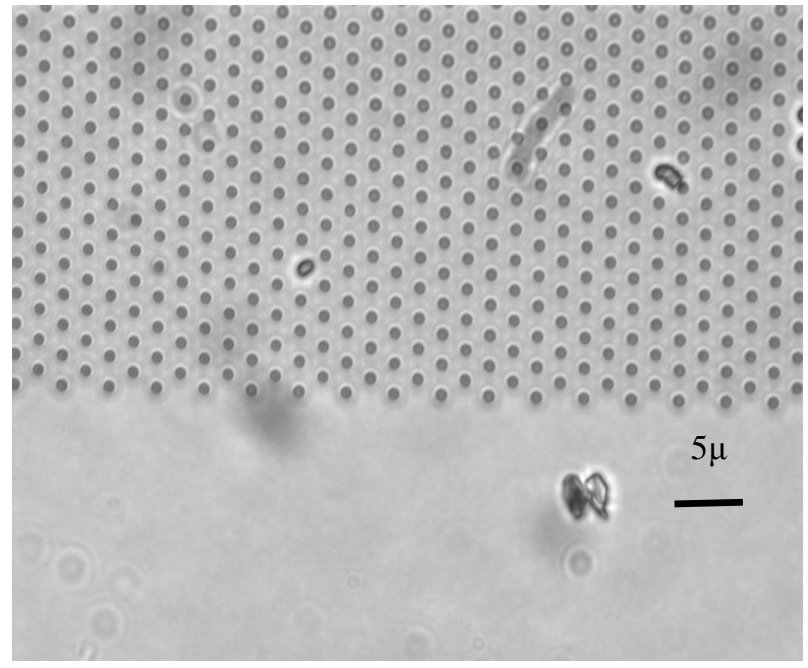

Figure 2. Microscope image of PDMS stamp (1 $1 \mu \mathrm{m}$ dots, $1 \mu \mathrm{m}$ spacing $)$

\section{Coverslips preparation}

A very thin glass, $100 \mu \mathrm{m}$, microscope coverslip substrate was cleaned and transferred to boiling water for $10 \mathrm{~min}$, prior to the silanisation procedure. The silanisation process tended to reduce the adsorption of protein polar compounds onto the surface of the glass substrate. Consequently possible reactions between the protein and the glass were eliminated.

The substrates were silanised by immersing them in a mixture of 1\% 3-aminopropyltrimethoxysilane (3-APS) in methanol: acetic acid, $99.9: 0.1$ by volume for $15 \mathrm{~min}$ after boiling[10]. Substrates were rinsed in methanol for $1 \mathrm{~min}$ then dried with nitrogen and baked at $120^{\circ} \mathrm{C}$ for 5 min to complete the hydrolysis reaction [12]. A $2.5 \%$ solution of glutaraldehyde in de-ionised water was used as a cross-linker and applied to the silanised substrates for 30min. Excess glutaraldehyde solution was removed by rinsing in the de-ionized water [10].

The first trial showed that the pattern definition and amount of transferred protein on the plane coverslip by far is less than the one on the pretreated coverslips.

\section{Stamp surface Plasma treatment}

Owing to the fact that unmodified PDMS presents a hydrophobic surface, the stamps were difficult to wet with the aqueous protein solution. The untreated, hydrophobic, PDMS is prone to the adsorption of other hydrophobic species and will often nucleate bubbles [13]. Exposure to air plasma alters the PDMS surface rendering it hydrophilic; however, this hydrophilic property of surface is unstable in air and the surface reverts to being hydrophobic in less than hour [13]. Fig 3 presents an illustration of the effect of surface plasma treatment. Without treatment, the liquid has a large contact angle with the stamp. The stamp features can behave in a superhydrophobic manner exacerbating the problem. With plasma treatment the contact angle is significantly reduced and the protein wets evenly to the stamp.

Fig 4. presents contact prints from stamps with and without surface plasma treatment. The treated stamp produces a 
uniform array of protein islands while the untreated stamp exhibits bubbles where the protein has failed to wet the stamp.

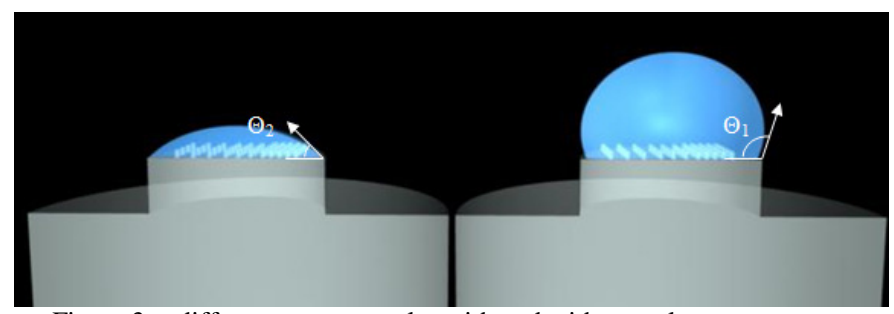

Figure 3. different contact angles with and withoug plasma treatment.

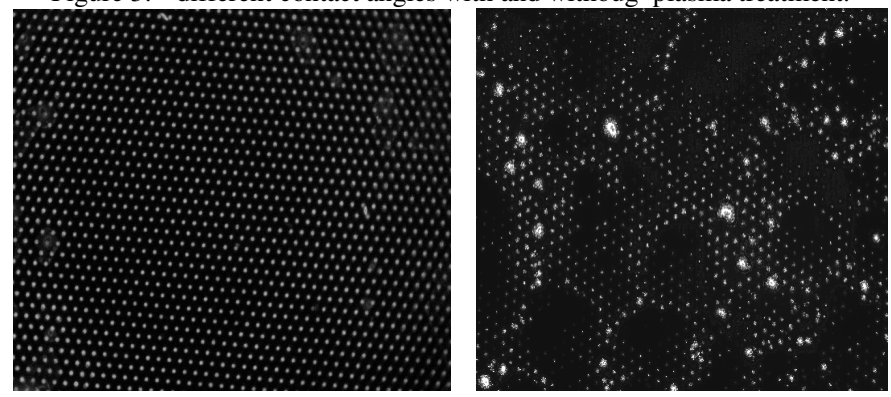

Figure 4. optical microscopy image of printed protein (Left ) with plasma treated stamp, and (Right) with unmodified stamp bubbles, place protein not being printed, can be found all over the picture..

\section{E. Protein array stamping}

The stamping procedure was carried out according to Branch et al. [10]. The cured PDMS stamp was adsorbed with FITC-labeled Fibrinogen solution (FITC-FGN, 100 $\mu \mathrm{g} / \mathrm{ml}$ water) by applying several droplets of protein on the stamp face and soaking the stamp in the protein for 2 hours. The stamp was then dried using nitrogen. Coverslip stamping was conducted using a mask aligner, which enabled visual monitoring of stamp deformation to regulate the force applied during the process. A glass coverslip $(60 \times 22 \mathrm{~mm})$ was suspended from a blank mask plate using a droplet of water. The stamp was brought to contact with the coverslip gradually. Contact was maintained for 2 minutes. Although a majority of protein is delivered within the first 10 seconds, the transfer is incomplete until 1 minute after contact [12].

\section{PROTEIN ARRAY STAMPING RESULTS}

After fabricating the stamp and transferring the protein as described in Section II, the transferred protein was examined using a standard optical microscope as well as fluorescence microscope. It is evident that distinct islands of protein have been formed. Microscope images of the transferred protein are presented in Fig 5 and Fig 6.

An initial application of these protein arrays to the investigation of blood platelet adhesion has been conducted. Fig. 7 presents an image of the spreading response of isolated platelets on the micropatterned surface. Preliminary findings are encouraging and suggest that unlike continuous fibrinogen substrates, modification of Fibrinogen surface distribution has a considerable impact on the geometry and overall behavior of platelet surface adhesion and spreading dynamics. Significantly, these initial experiments suggest that platelets are able to cantilever across protein free gaps and adhere and spread across multiple isolated protein islands (Fig. 7).

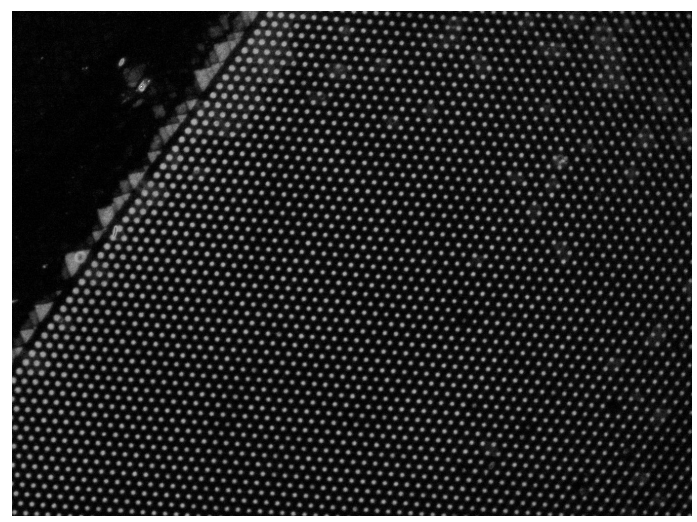

Figure 5. The optical microscopy image of printed protein $(1 \mu \mathrm{m}$ dots, with $1 \mu \mathrm{m}$ seperation)

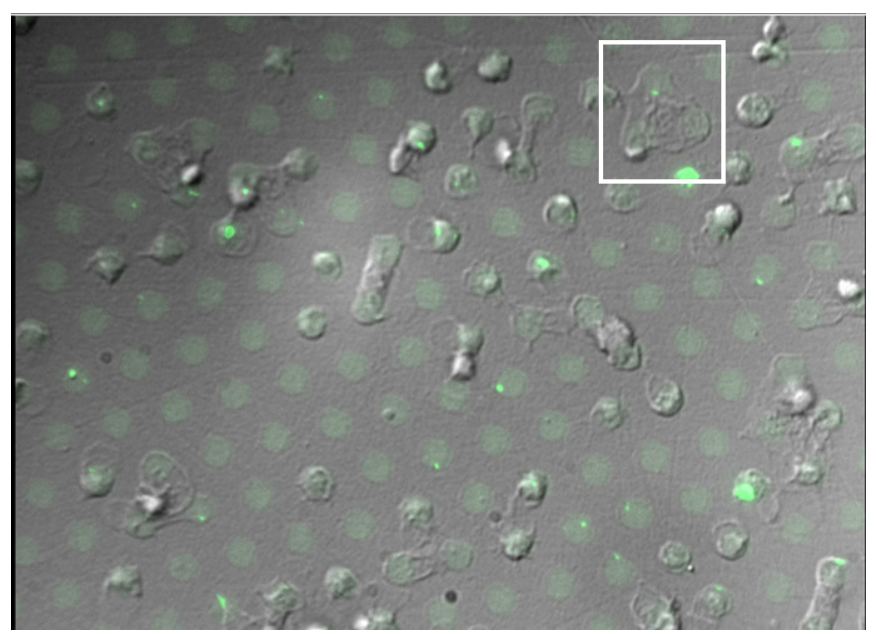

Figure 6. DIC \& fluorescent composite image of microprinted FITCFibrinogen ( $3 \mu \mathrm{m}$ island with $3 \mu \mathrm{m}$ seperation) [Green] showing the adhesion and spreading behaviour of isolated platelets

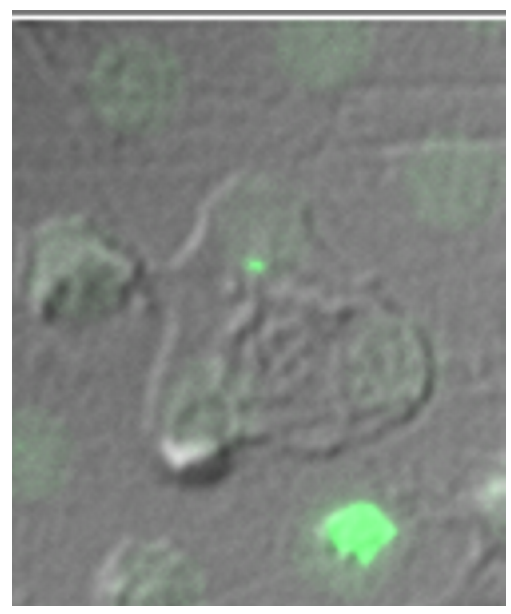

Figure 7. Zoomed image from Fig. 6 (White Box) demonstrating the ability of platelets to traverse multiple protein islands via the formation of bridging membrane extensions. 


\section{DISCUSSION}

We have shown fabrication of stamp and stamping of micron scale protein arrays has been achieved. Furthermore, an initial application of these protein arrays to the study of blood platelet adhesion has been conducted and the results suggest that detectable amounts of protein for cell are transferred from aqueous solution to the pretreated glass. The amount of transferred protein depends on several factors including the applied pressure on stamp, adsorption of protein to the stamp and then releasing from stamp, and the age of the stamp [6]. We find the pressure applied to the glass support of the stamp is crucial to have well defined and well separated islands of protein especially in the case that the stamp features are wide and shallow. Insufficient pressure could result in irreversible adsorption of protein onto stamp [12]; on the other hand excess pressure might result in squeezing, deformation of stamp, and transferring protein from regions between the raised features of the stamp. The optimum pressure can be determined by visual inspection of stamp during the process within the mask aligner. This should be just sufficient to make contact between the raised area of stamp and coverslip so that the protein can covalently react with pretreated coverslip.

The surface chemistry of the stamp is important for both adsorption of protein at the first instance and also releasing protein from the stamp onto the glass cover slip. It is possible to treat the stamp surface using a release layer (such as SDS) which can aid protein release [12], however, in this paper we have shown that the printed protein pattern improved by means of changing the PDMS stamp surface hydrophobic property to the hydrophilic using Air/Plasma treatment

Although the protein patterns were achieved, further investigations must be conducted to control the amount of protein transferred with this technique.

\section{REFERENCES}

[1] Nesbitt, W.S., Giuliano, S., Kulkarni, S., Dopheide, C.L., Harper, I.S., and Jackson, S.P. A Novel Inter-cellular Calcium Signalling Mechanism Driving Platelet Aggregation Under Flow. (2003) J. Cell Biol. 16 0(7): 1151-1161

[2] Nesbitt, W.S., Kulkarni, S., Giuliano, S., Goncalves, I., Dopheide, C.L., Harper, I.S., Salem, H.H., and Jackson, S.P. (2002) Distinct Calcium signaling Mechanisms Utilized by Platelet GPIb/V/IX and Integrin $\alpha_{\mathrm{IIb}} \beta_{3}$ to Regulate Adhesion Dynamics under Flow. J. Biol. Chem. 277: 29652972.

[3] Kulkarni, S., Nesbitt, W.S., Dopheide, S.M., Hughan, S.C., Harper, I.S., Jackson, S.P. (2004) Techniques to Examine Platelet Adhesive Interactions Under Flow. In Methods in Molecular Biology Vol. 272: Platelets and Megakaryocytes: Vol. 1: Functional Assays. J. Gibbins \& Mahaut Smith P. (Ed), Humana Press Inc., Totowa NJ.

[4] Ivanova, O. Y. and Margolis, L. B. (1973): 'The use of phospholipid film for shaping cell cultures,' Nature, 242, pp. 200-201

[5] Letourneau, P. (1975): 'Cell-to-substratum adhesion and guidance of axonal elongation,' Dev. Biol., 44, pp. 92-101

[6] Hammarback, J. A., Palm, S. L., Furcht, L. T. and Letourneau, P. C. (1985): 'Guidance of neurite outgrowth by pathways of substratumadsorbed laminin,' J. Neurosci. Res., 13, pp. 213-220

[7] Singhvi, R., Kumar, A., Lopez, G. P. Stephanopolous, G. N., Wang, D. I. C., Whitesides, G. M. and Iingber, D. E. (1994): 'Engineering cell shape and function,' Science, 264, pp. 696-698

[8] B. Michel et al. "Printing meets lithography", IBM J. Res. \& Dev. 45, 5 (2001) pp.697-719

[9] SOFT LITHOGRAPHY Younan Xia, George M. Whitesides Annual Review of Materials Science, August 1998, Vol. 28, Pages 153-184

[10] D.W. Branch, J.M. Corey, J.A. Weyhenmeyer, G.J. Brewer and B.C. Wheeler, Microstamp patterns of biomolecules for high-resolution neuronal networks. Med Biol Eng Comput 36 (1998), pp. 135-141

[11] L. Lauer, C. Klein and A. Offenhausser, Spot compliant neuronal networks by structure optimized micro-contact printing. Biomaterials $\mathbf{2 2}$ (2001), pp. 1925-1932

[12] John C.Chang, Gregory J.Brewer, Bruce C.Wheerler, An enhanced microstamping technique for controlled deposition of protein.

[13] J.Cooper McDonald, David C.Duffy, Jennelle R.Anderson, Daniel T.Chui, Hongkai Wu, Olivier J.A Schueller Fabrication of microfluidic systems in poly(dimethysioxane). 